\title{
Topological Analysis of Computed Three-Dimensional Viscous Flow Fields
}

\section{George S. Deiwert}

\author{
Gloghy ary \\ ㄴ. $2 \% 198 ?$ \\ LANGIEY RESEASCH CENTER \\ LIERARY, HASA \\ HARTONON YREIPIA
}


. 
NASA Technical Memorandum 84255

\section{Topological Analysis of Computed Three-Dimensional Viscous Flow Fields}

George S. Deiwert, Ames Research Center, Moffett Field, California 


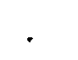


Topological Analysis of Computed Three-Dimensional Viscous Flow Fields

GEORGE S. DEIWERT

NASA Ames Research Center

Moffett Field, California

\section{Summary}

Computed solutions of the time-dependent, Reynolds-averaged Navier-stokes equations for three-dimensional flows having thin shear layers are analyzed, using topological concepts. Specific examples include the transonic flow over a body of revolution with conical afterbody at moderate angles of incidence to the free stream. Experimental flow-visualization techniques are simulated graphically to visualize the computed flow. Scalar and vector fluid dynamic properties, such as pressure, shear stress, and vorticity on the body surface, are presented as topological maps, and their relationship to one another in terms of orientation and singular points is discussed. The extrapolation from these surface topologies toward the understanding of external flow-field behavior is discussed and demonstrated.

Introduction

Computational fluid dynamics has progressed in recent years such that with present numerical algorithms and modern computational facilities it is becoming feasible to simulate complex viscous flows over three-dimensional configurations of practical interest. Typical solutions are obtained in the form of very large data sets, of the order of millions of words, that define the flow-field properties at a large number of discrete points on and about the body of interest. To visualize flow-field features, techniques devised and used by experimentalists can be emulated, and graphic displays can be generated that yield comparable information. Experimental visualization techniques include surface-oil-streak patterns, shadowgraphs, schlieren photographs, interferograms, holograms, tufts, dye, smoke, vapor screens, and hydrogen bubbles. In addition, flow fields are probed for quantitative information with tubes, wires, films, and light beams. Similar quantitative 
information can be extracted from computed data bases in a straightforward manner.

Particular information concerning complex three-dimensional flow features is found in the flow-field topology on or very near the surface. This topology is the foundation upon which the external flow rests, and an understanding of the surface features can provide substantial insight into the behavior of the rest of the flow field. The analysis by squire [1] shows that surface oil-flow visualizations closely approximate the skin-friction lines and limiting streamlines. Some considerable analyses by Legendre [2], Lighthill [3], Perry and Fairlie [4], Hunt et al. [5], and Tobak and Peake [6] provide a mathematical basis that permits detailed descriptions of three-dimensional separation and reattachment patterns based on surface vector patterns and their singular points. By extracting surface or near-surface vector and scalar topologies from discretized computed three-dimensional flow fields, the computational fluid dynamicist can use these mathematical concepts to analyze regions of separation and reattachment and to relate computed patterns to experimental observations.

To illustrate some of the topological principles for computed data bases and to relate the behavior of various vector and scalar topological patterns to each other, a series of computed results for flow over a conical afterbody at moderate angles of incidence to the free stream is considered.

The thin-shear-layer approximation to the time-dependent, three-dimensional, compressible, Reynolds-averaged NavierStokes equations was solved numerically, using the implicit finite-difference method of Beam and warming [7]. The computations correspond to a series of experiments by shrewsbury [8] for flow over a pointed cylindrical forebody of caliber nine followed by a $15^{\circ}$ conical afterbody and a two-thirds-forebodydiameter cylindrical sting. The flow conditions correspond to a free-stream Mach number of 0.9 and a Reynolds number based on forebody diameter of nearly $3 \times 10^{6}$. Steady-state solutions were computed for angles of incidence to the free stream of $0^{\circ}, 2^{\circ}, 4^{\circ}, 6^{\circ}, 8^{\circ}$, and $12^{\circ}$. Quantitative comparisons of 
computed and experimentally measured surface pressure data for angles of incidence up to $6^{\circ}$ have been reported by Deiwert [9] and good agreement was found. Computed results for the $8^{\circ}$ and $12^{\circ}$ cases will be used as illustrative examples here.

Discussion

To relate various surface properties, the incompressible Navier-Stokes equations are written for the surface where all velocity components are zero and $\omega_{\zeta}=0$ :

$$
\mathrm{p}_{\xi}=\mu \mathrm{u}_{\zeta \zeta}=\mu\left(\omega_{\eta}\right)_{\zeta}
$$

and

$$
\mathrm{p}_{\eta}=\mu \mathrm{v}_{\zeta \zeta}=\mu\left(\omega_{\xi}\right)_{\zeta},
$$

where $\xi$ and $n$ are coordinates in the plane of the body surface and $\zeta$ is normal to the surface. The above relations tell us that the tangential vorticity is aligned in the direction of surface isobars. For a small distance $\zeta$ from the surface, the velocity vector field in the plane parallel to the surface is given by

$$
\vec{v}=\left(\vec{w}_{w} \times \vec{n}\right) \zeta=\vec{\tau}_{w} \zeta / \mu
$$

where $\vec{\omega}_{w}$ is the vorticity vector at the surface and $\vec{n}$ is the unit surface normal vector. This relation tells us that $\vec{\tau}_{w}$ (surface shear) is orthogonal to $\vec{\omega}_{w}$ (tangential vorticity) and hence to the direction of th s surface isobars.

Discretized solutions obtained from finite-difference equations do not provide detailed descriptions of behavior at singular points. Small-perturbation analyses of the differential equations in the vicinity of singular points can provide more detail and enhance our understanding of the flow behavior in these critical regions, which occur when the flow separates and reattaches. Two types of separation typically occur in three-dimensional flows: one when the components of tangential vorticity approach zero simultaneously, and the other when there is a convergence of surface streamlines. In the latter type of separation we can see from continuity that for flow through a rectangular stream tube whose base $b$, is 
the distance between two adjacent limiting streamlines and whose variable height is $\zeta$,

$$
\left|\vec{\omega}_{w}\right| \zeta^{2} \mathrm{~b} / 2=\text { constant }
$$

Thus, when surface streamlines converge, $b$ decreases and there is a corresponding increase in $\zeta$ and a "piling up" of stream tubes that results in three-dimensional separation in the form of a vortex sheet. In the former type separation $\left(\omega_{w} \rightarrow 0\right)$, small-perturbation analysis suggests that in the vicinity of a singular point there is also a pressure extremum which can be defined by $\underline{p}_{\xi}=p_{\eta}=0$, where $\xi$ and $\eta$ are mutually orthogonal.

For the present cases, limiting streamlines were determined by tracing particle paths parallel to the body surface (neglecting the surface normal velocity component) at a very small distance from the surface $(\zeta / d=0.00008)$. These lines are identical to skin-friction lines and, according to squire [1], should closely approximate oil-streak patterns. Shown in Fig. 1 are side views of limiting streamline patterns over the complete configuration at angles of incidence of $8^{\circ}$ (Fig. Ia) and $12^{\circ}$ (Fig. 1b). In both cases the streamlines on the cylindrical body strongly converge near the generator, $45^{\circ}$ from the lee generator, indicating a three-dimensional separation in the form of a vortex sheet. Immediately aft of the cylinderical-forebody/conical-afterbody junction, the streamlines turn abruptly and completely circumscribe the afterbody. This again is an indication of threedimensional separation and will be described in more detail subsequently. Just aft of the conical-afterbody/cylindricalsting junction, the flow reattaches along a line circumscribing the sting, and the streamlines proceed to converge downstream along a lee generator of the sting.

Shown in Fig. 2 are surface topologies of several fluid dynamic properties for the afterbody at $8^{\circ}$ incidence to the free stream. Included are lee, lateral, and windward views showing lines of surface shear (solid) with lines for $p_{\xi}=0$ and $\mathrm{p}_{\eta}=0$ (dashed) superposed, where $\xi$ and $n$ are the axial 
and circumferential directions, respectively. Just aft of the forebody/afterbody junction there is a line of separation that completely circumscribes the afterbody. This line begins at a separation saddle point on the lee generator and terminates at a separation node on the windward generator. Immediately upstream of this separation line is a line of $p_{\xi}=0$ (dashed) which intersects $\mathrm{p}_{n}=0$ lines coincident with the lee and windward generators and $p_{\eta}=0$ lines a few degrees to either side of each generator. All saddle and node points are denoted by symbols and are defined in the figure captions. On the lee side, the pressure extrema are of the form saddle-nodesaddle and correspond to the separation saddle point indicated by the limiting streamlines on the lee generator. On the windward side, the pressure extrema are of the form node-saddlenode and correspond to the separation node indicated by the limiting streamlines on the wind generator. Aft of the afterbody/sting junction there is a line of reattachment completely circumscribing the sting. There are reattachment nodes on both the lee and windward generators and a reattachment saddle $60^{\circ}$ from the lee generator, as indicated by the limiting streamlines. Immediately downstream of the line of reattachment is the dashed line of $\mathrm{p}_{\xi}=0$, which intersects $\mathrm{p}_{n}=0$ lines coinciajent with the lee and windward generators and the $p_{\eta}=0$ line at $83^{\circ}$ from the lee generator. These points are node, node, and saddle, respectively, and correspond to the reattachment singular points directly. Between the line of separation and the line of reattachment, the flow over the surface is reversed and a separation bubble exists. The directions of the limiting streamlines under the bubble indicate counterclockwise circulation when seen in the lateral view. Downstream of reattachment there is a strong convergence of streamlines about $55^{\circ}$ from the lee generator, indicating separation of the second kind.

Shown in Fig. 3 is a lateral perspective of the afterbody at $3^{\circ}$ incidence. Figure $3 a$ is a surface pressure map, in which $C_{p}$ is shown as a function of axial distance ( $\xi$ ) and circumferential angle $(\phi)$ from the lee generator. Surface pressure contours (dashed) are mapped on the afterbody in Fig. $3 b$ and are superposed over the limiting streamlines to illustrate 
regions of orthogonality. The sonic surface envelope over the afterbody is shown in Fig. 3c, with skin-friction lines drawn on the afterbody surface. It is the termination of the supersonic flow inside the sonic surface by means of a threedimensional shock front that induces flow separation on the afterbody near the forebody/afterbody junction. Included also in Fig. $3 c$ is a dashed line for $\left(\vec{w}_{w} \cdot \vec{n}\right)=0$, which is aligned with the vortex sheet that separates from the sting. There are also zero vorticity lines near both the lee and windward generators that cross these generators at the singular points indicated in the limiting streamline patterns.

As the angle of incidence is increased, the complexity of the surface topology can increase. Shown in Fig. 4 are lee, lateral, and windward views for the afterbody at $12^{\circ}$ incidence. As observed in the $8^{\circ}$ solution, there is a line of separation aft of the forebody/afterbody junction and a line of reattachment aft of the afterbody/sting junction. Both lines completely circumscribe the body. Beginning at the intersection of the separation line and the lee generator and progressing along the separation line to the windward generator, there is a series of singular points as follows: node-saddle-nodesaddle-node-saddle at $\phi=0^{\circ}, 22^{\circ}, 67^{\circ}, 102^{\circ}, 160^{\circ}$, and $180^{\circ}$, respectively. These points are preceded in the streamwise direction by pressure extrema at $\phi=0^{\circ}, 30^{\circ}, 145^{\circ}$, and $180^{\circ}$, which are node-saddle-node-saddle points, respectively. It is of particular interest to note that there exist more singular points on the line of separation than there are pressure extrema in the vicinity. The "extra" points appear to be the separation node and saddle at $\phi=67^{\circ}$ and $102^{\circ}$, respectively. In addition, the flow pattern under the separation bubble is broken into several distinct counterrotating zones, with the most prominent discriminator being a line connecting the separation saddle at $\phi=102^{\circ}$ and the reattachment saddle at $\phi=75^{\circ}$. Along this line there is strong convergence of the limiting streamlines, indicating a prominent bubble bifurcation by means of a vortex sheet. The complex three-dimensional flow behavior in the separation bubble obviates a simpler 
surface analysis and a one-to-one correspondence between pressure extrema and singular points in the surface pattern.

Along the line of reattachment there are nodal points on both the lee and windward generators and a saddle $75^{\circ}$ from the lee. These are accompanied by pressure extrema slightly downstream with nodal points on both the lee and windward generators and a saddle-node-saddle cluster at $\phi=65^{\circ}, 75^{\circ}$, and $97^{\circ}$, respectively, near the reattachment saddle. Again, as in the $8^{\circ}$ case, convergence of streamlines on the sting near the $\phi=70^{\circ}$ generator indicates the separation of a vortex sheet.

Shown in Fig. 5 are perspective views of the $12^{\circ}$ solution. Figure $5 a$ shows the surface pressure map, $C_{p}(\xi, \phi)$. Dashed surface pressure contours are superposed on the surface streamline pattern in Fig. 5b. The sonic surface and surface streamlines are included in Fig. $5 \mathrm{c}$, with a dashed line for $\left(\vec{\omega}_{\mathrm{w}} \cdot \vec{n}\right)=0$ superposed. This zero vorticity line is coaligned with the location of converged surface streamlines. Other zero vorticity lines appear near the lee and windward generators, crossing these lines at the separation and reattachment saddles and nodes.

\section{Concluding Remarks}

In the examples discussed above, we have seen that in regions of three-dimensional separation where the surface vorticity goes to zero $\left(\vec{\omega}_{\mathrm{w}} \cdot \overrightarrow{\mathrm{n}} \rightarrow 0\right)$, the singular points defining the lines of separation are preceded by accompanying pressure extrema of like character (i.e., node:node and saddle:saddle) and singular points defining lines of reattachment precede accompanying pressure extrema of like character (note that clusters of singular points behave as a singular point so that a node-saddle-node behaves as a node and a saddle-nodesaddle behaves as a saddle). For the $8^{\circ}$ case, there is a direct correspondence between the pressure extrema and the singular points of separation and reattachment. For the $12^{\circ}$ case, where the flow pattern is more complex, more singular points were observed along the separation line than were suggested by accompanying pressure extrema. In both cases $\left(8^{\circ}\right.$ and $\left.12^{\circ}\right)$ singular points are indicated both by the 
limiting streamlines and by zero surface vorticity. For the separated vortex sheet developing from the reattachment saddle there is an accompanying line of zero surface vorticity coaligned with the converging limiting streamlines.

\section{References}

1. Squire, L. C.: The motion of a thin oil sheet under the boundary layer on a body. RAE Aero 2636, Feb. 1960.

2. Legendre, R.: Separation de l'ecoulement laminaire tridimensionnel. Rech. Aero. 54 (1956) 3-8.

3. Lighthill, M. J.: Attachment and separation in threedimensional flow. In Laminar Boundary Layers, L. Rosenhead, II (ed.). Oxford U. Press, 1963.

4. Perry, A. E. and Fairlie, B. D.: Critical points in flow patterns. In Advances in Geophysics, New York: Academic Press, 1974.

5. Hunt, J. C. R.; Abell, C. J.; Peterka, J. A.; and Woo, H.: Kinematical studies of the flows around free or surfacemounted obstacles: applying topology to flow visualization. J. Fluid Mech. 86 (1978) 179-200.

6. Tobak, M. and Peak, D. J.: Topology of three-dimensional separated flows. Ann. Rev. Fluid Mech. I4 (1982) 61-85.

7. Beam, R.; and Warming, R. F.: An implicit finitedifference algorithm for hyperbolic systems in conservation-law-form. J. Comp. Phys., 22, Sept. (1976).

8. Shrewsbury, G. D.: Effect of boattail juncture shape on pressure drag coefficients of isolated afterbodies. NASA TM X-1517, 1968.

9. Deiwert, G. S.: Numerical simulation of three-dimensional boattail afterbody flowfields. AIAA J., 19 (5), May (1981).
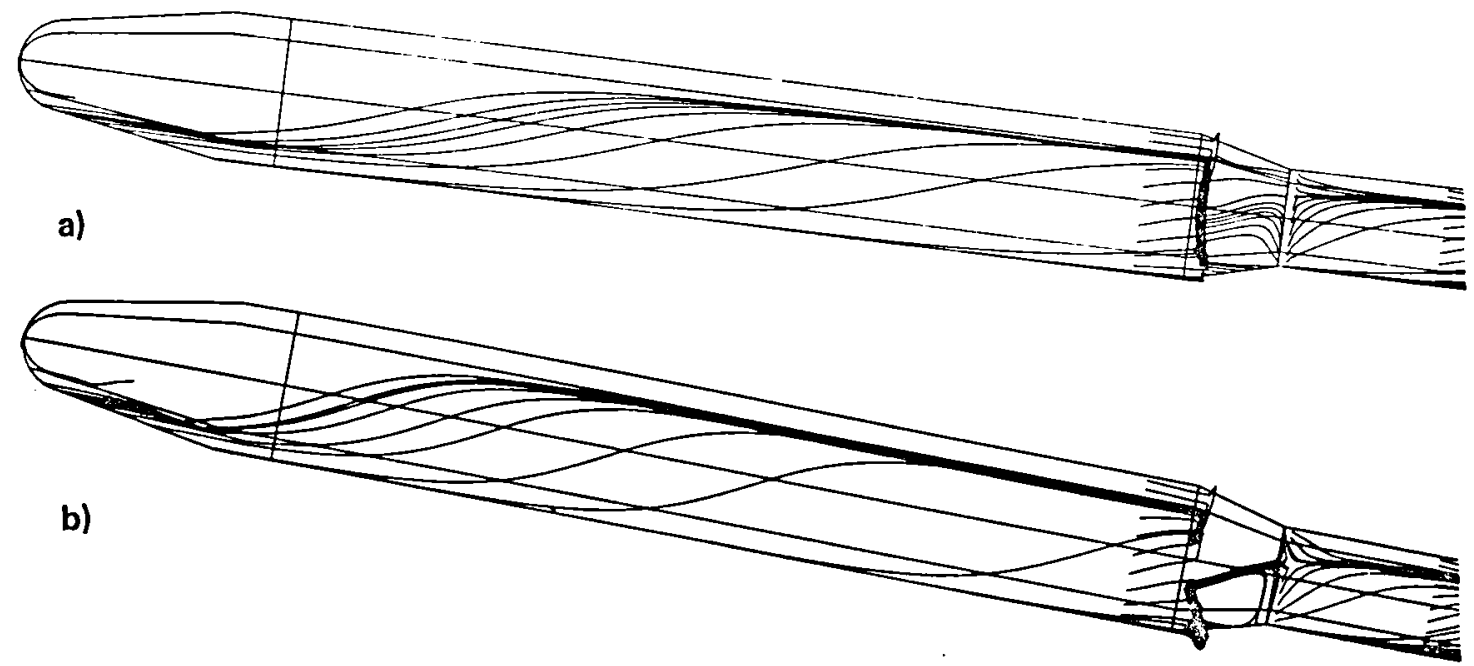

Fig. 1. Limiting streamline pattern on complete afterbody model at incidence $(\alpha)$ to the free stream (lateral view) $\mathrm{M}_{\infty}=0.9, \operatorname{Re}_{\mathrm{d}}=3 \times 10^{6}:$ (a) $\alpha=8^{\circ} ;$ (b) $\alpha=12^{\circ}$. 


\section{SHEAR GRADIENT}

$\begin{array}{ccc}0 & - \text { SADDLE - } & \Delta \\ \square & - \text { NODE - } & 0\end{array}$

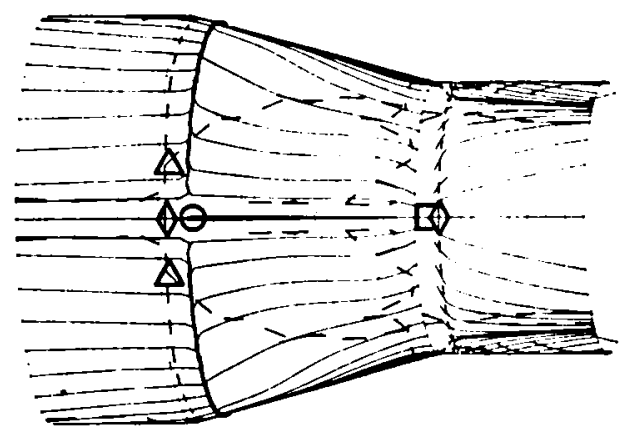

a)

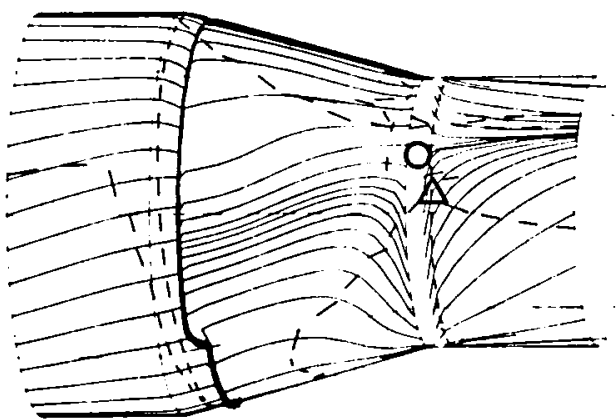

b)

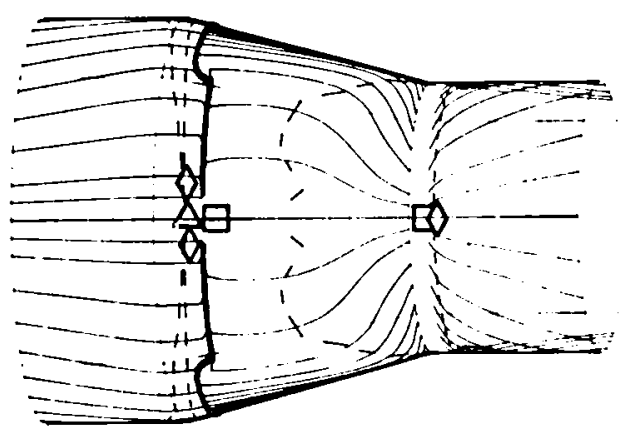

c)

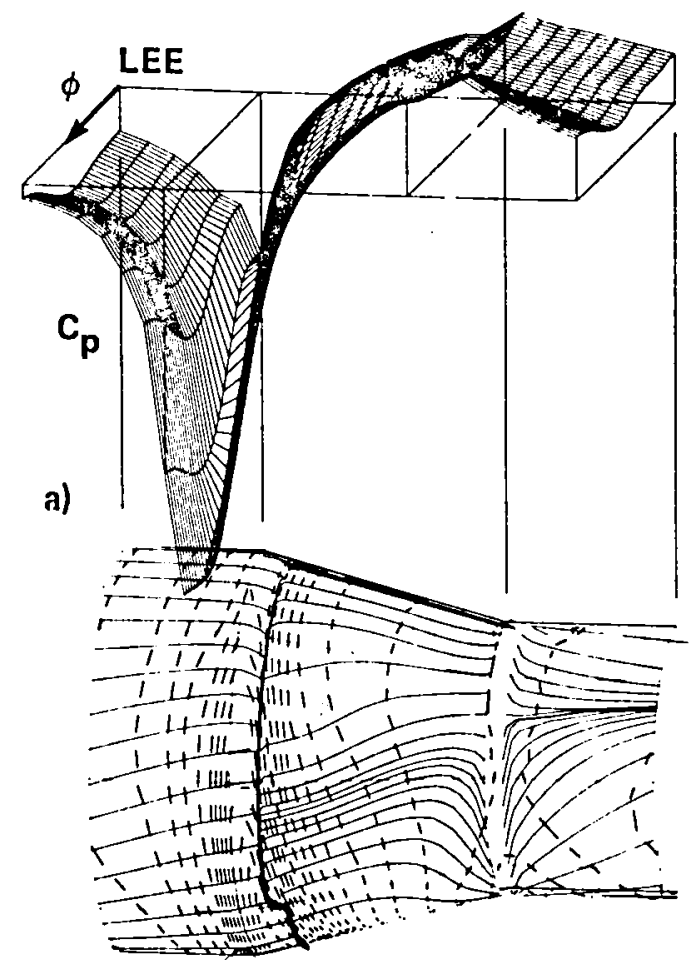

b)

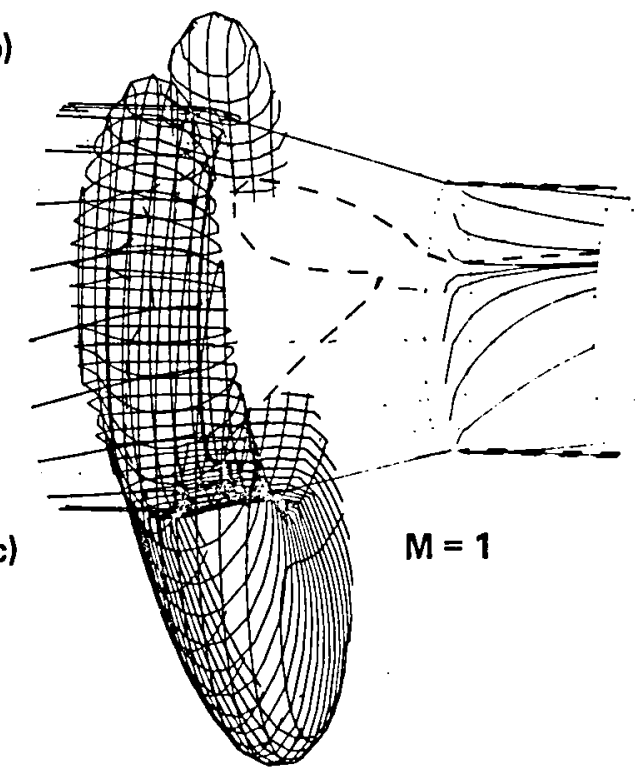

Fig. 3. Lateral perspective of flow features for conical afterbody at $8^{\circ}$ incidence, $M_{\infty}=0.9:$ (a) surface pressure; (b) isobars (--) and surface shear (-); (c) sonic surface, surface shear (-) and zero vorticity $\left(\vec{\omega}_{w} \cdot \vec{n}=0\right)(--)$.
Fig. 2. Surface shear (-) and zero pressure gradient (-) topology for conical afterbody at $8^{\circ}$ incidence, $M_{\infty}=0.9$ : (a) lee; (b) lateral; (c) windward side. 


$$
\begin{array}{cc} 
& \text { PRESSURE } \\
\text { SHEAR } & \text { GRADIENT } \\
0-\text { SADDLE }-\triangle \\
\square-\text { NODE }-\diamond
\end{array}
$$

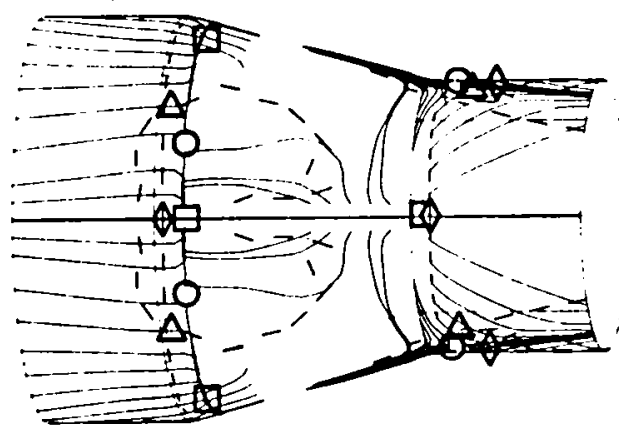

a)

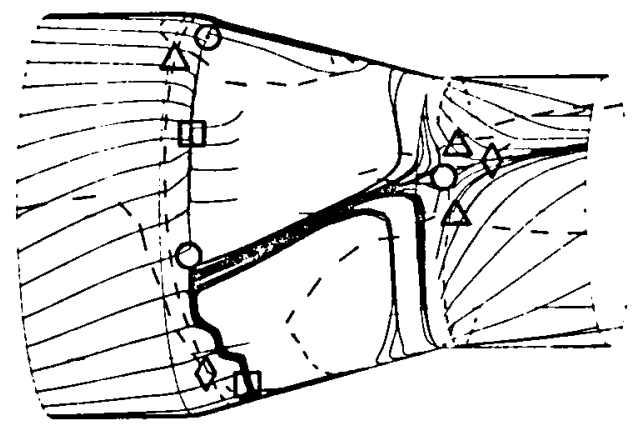

b)

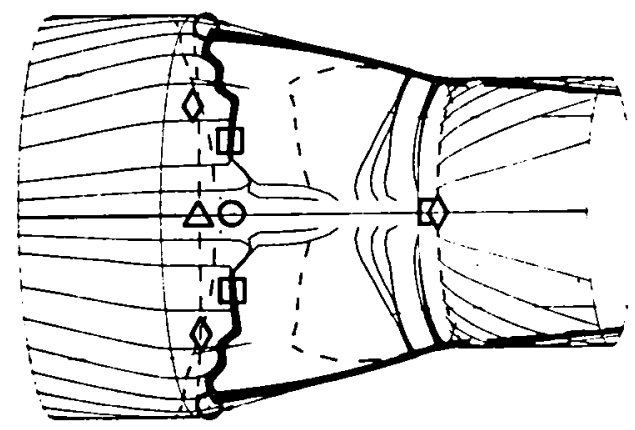

c)

Fig. 4. Surface shear (-) and zero pressure gradient topology (--), for conical afterbody at $12^{\circ}$ incidence, $\mathrm{M}_{\infty}=0.9$ : (a) lee; (b)

lateral; (c) windward side.
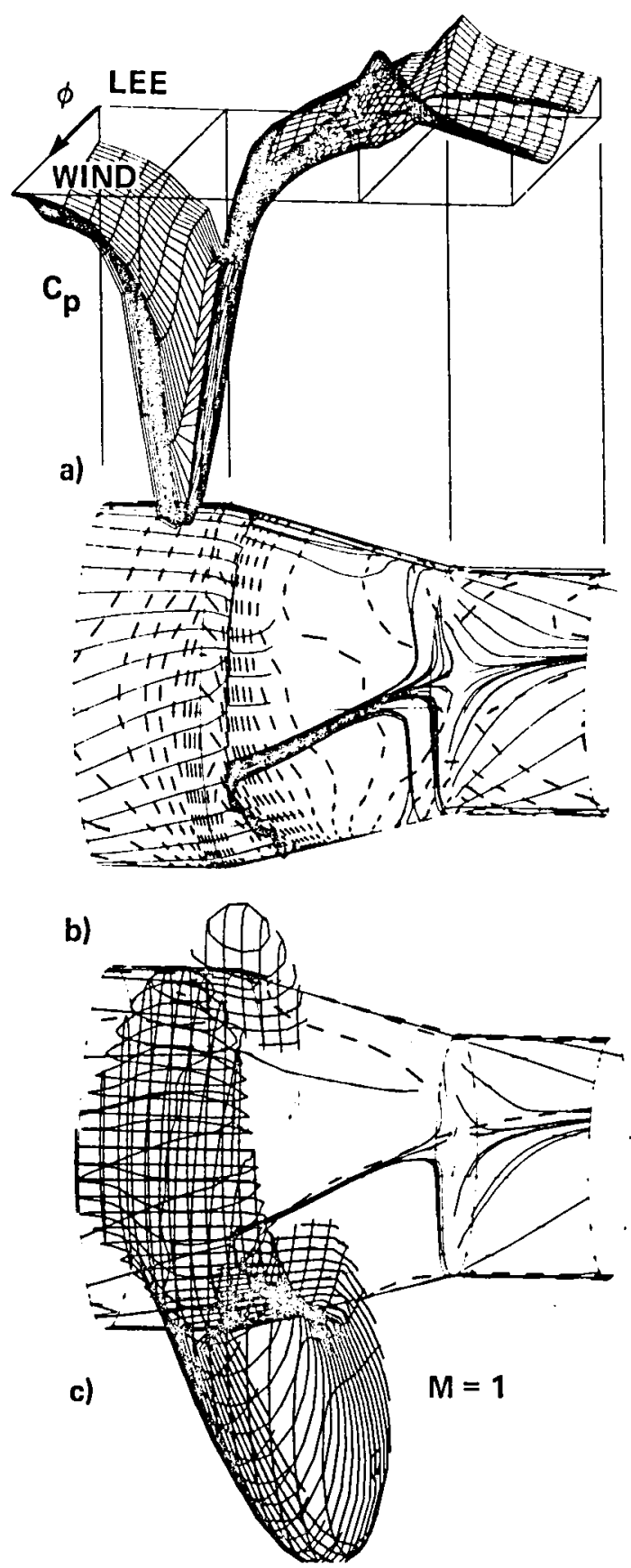

Fig. 5. Lateral perspective of flow features for conical afterbody at $12^{\circ}$ incidence, $\mathrm{M}_{\infty}=0.9:$ (a) surface pressure (b) isobars (--) and surface shear (-); (c) sonic surface, surface shear $(-)$ and zero vorticity $\left(\vec{w}_{W} \cdot \vec{n}=0\right)(--)$. 


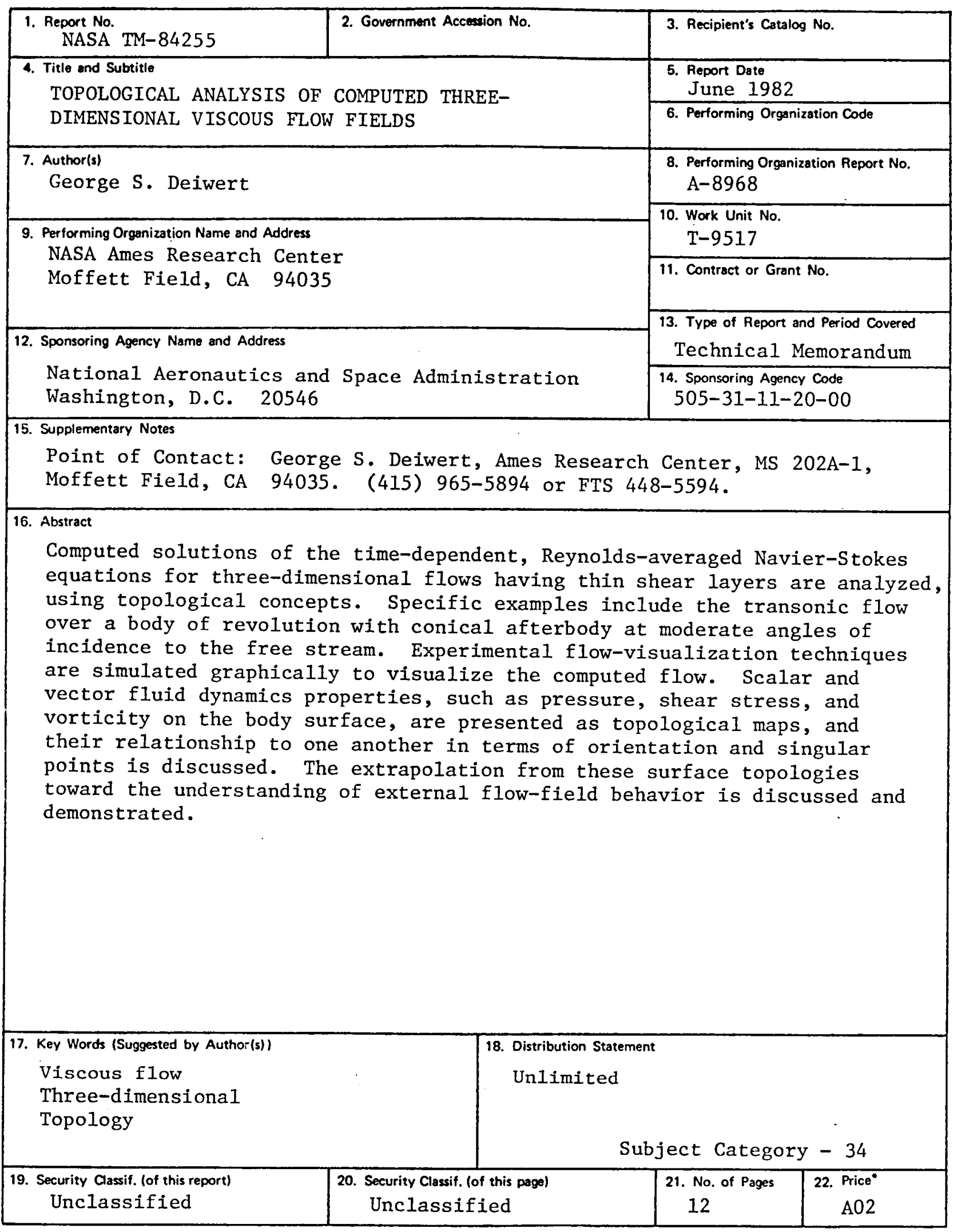

-For sale by the National Technical Information Service, Springfield, Virginia 22161 
. 



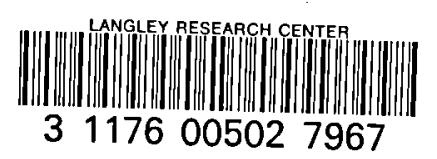

